\title{
DJ-1 Activation Raf/ERK Pathways Promotes Autophagy Maturation of PC-12 Cells
}

\author{
Xuezhong Li, Kun Zhao, Yuansu Zhuang, Xiaopeng Chen, Yi Liu \\ Jiangsu University Affiliated People's Hospital, Zhenjiang, China \\ Email: sudalxz@163.com
}

How to cite this paper: Li, X.Z., Zhao, K., Zhuang, Y.S., Chen, X.P. and Liu, Y. (2021) DJ-1 Activation Raf/ERK Pathways Promotes Autophagy Maturation of PC-12 Cells. Advances in Parkinson's Disease, 10, 1-13. https://doi.org/10.4236/apd.2021.101001

Received: January 20, 2021

Accepted: February 24, 2021

Published: February 27, 2021

Copyright (c) 2021 by author(s) and Scientific Research Publishing Inc. This work is licensed under the Creative Commons Attribution International License (CC BY 4.0).

http://creativecommons.org/licenses/by/4.0/

(c) (i) Open Access

\begin{abstract}
Park 7 gene encodes a conserved protein called DJ-1 protein, which involves autophagy stress, but the mechanism is unclear. Therefore, it is necessary to explore the mechanism of DJ-1 regulation PC-12 autophagical stress. Using CRISPR/Cas9 technique to construct DJ-1 knockout PC-12 cell lines, we culture wild-type and DJ-1 knockout PC-12 cell lines, establish oxidative stress cell model by $\mathrm{MPP}^{+}$, and divide them into wild-type control group (WT), wild-type intervention group $\left(\mathrm{WT}+\mathrm{MPP}^{+}\right)$, DJ-1 knockout control group $(\mathrm{KO})$ and $\mathrm{DJ}-1$ knockout intervention group $\left(\mathrm{KO}+\mathrm{MPP}^{+}\right)$, and explore the role of DJ-1 in regulating neuronal autophagy stress by cell viability assay, immunofluorescence, confocal, western blotting and electron microscopy. The results show that the growth ability of DJ-1 knockout cells is inferior to that of normal cells, and DJ-1 knockout cells are more sensitive to oxidative stress and more vulnerable to damage than wild-type cells. Exposing to $\mathrm{MPP}^{+}$, DJ-1 proteins undergo oxidative responses at Cys-106 sites, while DJ-1 knockout PC-12 cells do not show similar responses. The wild-type PC-12 cells have the confocal in both anti-oxidant DJ-1 antibody and anti-C-Raf phosphorylation antibody. The activated DJ-1 induces the phosphorylation of C-Raf at Ser338 sites to activate directly C-Raf, and subsequently activates ERK1/2 signaling pathways to antagonize $\mathrm{MPP}^{+}$-induced neurotoxicity. Lack of DJ-1, oxidative stress can not promote C-Raf activation. Although the phosphorylation level of cell ERK is also increased, the increase of intranucleus pERK is not obvious. Wild type and DJ-1 knockout PC-12 cells can produce autophagical stress in the face of oxidative stress, but the proportion of autophagolysosomes produced in wild type PC-12 cells is larger than that in DJ-1 knockout cells. PD98059 can reduce autophagy stress in the state of oxidative stress in wild-type PC-12 cells, and the number of autophagolysosomes is similarly reduced, while sorafenib decreased slightly DJ-1 the autophagical stress, and the proportion of autophagolysosomes decreased more. Therefore, we can infer that activated DJ-1 directly phosphorylates C-Raf at
\end{abstract}


Ser-338 sites, then activating C-Raf, subsequent activation of the MEK/ERK pathway. DJ-1 promotes autophagy maturation through the C-Raf/ERK pathway, thereby improving cell survival.

\section{Keywords}

DJ-1, C-Raf, ERK, Parkinson's Disease, Autophagical Maturation

\section{Introduction}

Park 7 gene encodes a conserved protein called DJ-1, and is found to have an autosomal recessive gene deletion mutation in 2003, which can lead to early onset Parkinson's disease (PD) [1]. DJ-1 consists of 189 amino acids, containing three cysteine residues at sites 46, 53 and 106. Of the three cysteine residues, the 106-site cysteine residues are highly sensitive to oxidative stress [2] [3] [4]. DJ-1 can bind directly to the C-Raf kinase domain rather than RAS, and Cys-106 mutants of DJ-1 show weaker binding capacity than wild-type [5] [6]. The trigger of autophagy is precisely regulated by a series of waterfall events, including MAPK/ ERK and p38 signaling pathways. ERK nuclear translocation is associated with autophagy stress [7] [8] [9], but the mechanism of DJ-1 genes regulating autophagy stress is still not completely clear, especially the effect on autophagy maturation has not been reported, so it is necessary to explore deeply.

\section{Materials and Methods}

\subsection{Knockout of DJ-1 Genes in PC-12 Cell Lines}

Using CRISPR/Cas9 techniques in PC-12 cells, we first designed a sg sequence in DJ-1 functional exon coding regions that could guide Cas9 cleavage, and cloned it into plasmids that express both sgRNA and Cas9 simultaneously. Then the constructed plasmid was transiently transfected into PC12 cells, and the expressed Cas 9 was directed to the target region by sgRNA, then the functional exon coding region DJ-1 was cut. The cells will cause frameshift mutation in the process of NHEJ repair, then DJ-1 genes was knock out in the PC12 cells. Finally, the cells will be cloned by finite dilution method to complete the screening of monoclonal cell lines and obtain completely consistent DJ-1 knockout PC12 cell lines, which was called as A12 cell line. A12 genotype was detected to have $25 \mathrm{bp}$ base loss in exon 3, A12: TGCAGTGTAGCCGTG-TCTGGAAGAAGCAA-25 $\mathrm{bp}$, in line with the goal of DJ-1 knockout.

\subsection{Cell Culture and Treatments}

We thaw the preserved wild-type PC12 and DJ-1 knockout PC-12 cells, and incubate them in a incubator for $2 \mathrm{~d}$. Then the cells are collected by trypsin digestion, inoculated in $75 \mathrm{~cm}^{2}$ culture bottles at a density of $2 \times 10^{6} /$ flask. They are incubated with a DMEM containing 10\%FBS for 2 days, then digested by trypsin 
and collected for experiment. The cells are cultured in fresh DMEM for $3 \mathrm{~h}$ in all experiments, supplemented with $0.1 \%$ bovine serum albumin (BSA, A7030, Sigma). We intervene them with $\mathrm{MPP}^{+}(5 \mu \mathrm{g} / \mathrm{mL}$; Sigma), Raf/MEK/ERK signaling pathway blocker sorafenib ( $5 \mu \mathrm{mol} / \mathrm{L}$, Med Chem Express) and ERK signaling pathway blocker PD98059 (10 umol/L; Sigma), respectively or in combination. Then the cells are collected for further experiments, such as the effects of DJ-1 on cell viability, C-Raf activation, pERK nuclear translocation and autophagy stress, etc.

\subsection{Immunofluorescence Staining and Confocal}

Immunofluorescence staining is based on the literature [10]. The cells collected in the experiment are fixed at $4^{\circ} \mathrm{C}$ with $4 \%$ paraformaldehyde for $15 \mathrm{~min}$, with $0.1 \%$ triton $\mathrm{X}-100$ for $10 \mathrm{~min}$ at room temperature, and then react with primary antibody at $37^{\circ} \mathrm{C}$ for $3 \mathrm{~h}$. Here, primary antibodies contain mouse anti pERK (1:1000; chemicon), mouse anti C-Raf monoclonal (1:50, biorbyt), rabbit anti DJ-1 polyclonal (1:50, abcam) or rabbit antioxidant DJ-1 monoclonal (1:50, ab7608, abcam) antibodies. Subsequently incubation is performed at room temperature with a secondary antibody (anti-mouse or rabbit cy 3 complement receptor (1:300) or anti-mouse cy2 monoclonal antibody (1:200; sigma) for 1 hour). The cells are then stained with DAPI (1:1000; sigma) and observed. Before each protocol, cells are rinsed 3 times with $0.01 \mathrm{M}$ PBS for 5 minutes each time. Then fluorescence microscope and confocal microscope are used to observe, and results are obtained by two independent individuals in a blind way.

\subsection{Western Blotting}

Cell lysates are prepared in RIPA lysis buffer, and western blotting as described in the literature [11]. Nuclear protein is extracted according to nucleoprotein extraction kit instructions. The antibodies used include anti-C-Raf (1:500, Biorbyt), anti-phosphorylation-C-Raf (Ser-338) (1:500, Cell Signaling Technology), anti-C-Raf (Ser259) (1:500, Cell Signaling Technology), anti-ERK1/2 (1:500, Cell Signaling), and anti-phosphorylation ERK1/2 (1:500, Cell Signaling Technology) antibodies. Proteins are quantified by automated radiation analysis by density measurement (GS-700 imaging densitometer, Bio-Rad).

\subsection{Electron Microscopy}

PC-12 cells ultrastructural changes, especially the changes of autophagy-related organelles, were analyzed by transmission electron microscopy see the literature for details [12]. We used transmission electron microscope (Hitachi, Japan $\mathrm{H}-7500$, Suzhou University) to analyze the characteristics of autophagy vesicles by morphological characteristics. Autophagosomes contained intact cytoplasminto early stage, and partially disintegrating and electron dense in late stage, while autophagolysosomes were the products of autophagy and lysosome binding [8]. We randomly chose 10 micrographs (primary magnification, 10,000) 
from each sample to count the number of autophagosomes and autophagolysosomes in intact cells, respectively. Then the proportion of autophagosomes and autophagyolysosomes was analyzed.

\subsection{Statistical Analysis}

All data were collected from at least three independent experiments in triplicate. Through Student $t$ test or single factor ANOVA and Bonferroni multiple comparison test, the significance of the influence between control conditions and experimental conditions was determined. Statistical significance was set to $\mathrm{p} \leq$ 0.05 .

\section{Results}

The growth ability of DJ-1 knockout cells is significantly lower than that of wild-type PC-12 cells, which is more sensitive to oxidative stress.

Literature studies have shown that DJ-1 plays a regulatory role in cell growth and proliferation, while oxidative stress has a negative effect on them [4] [13] [14] [15], so it is necessary to observe the effect of DJ-1, oxidative stress or both on cell viability. In this study, we observe the effect of various interventions on cell viability by MTT detection (Figure 1). The results show that the OD values of the cells in the intervention groups are significantly lower than that in the control groups, and the cells in the DJ-1 knockout groups are lower than those in the wild type cells, which is statistical differences. DJ-1 knockout cells are

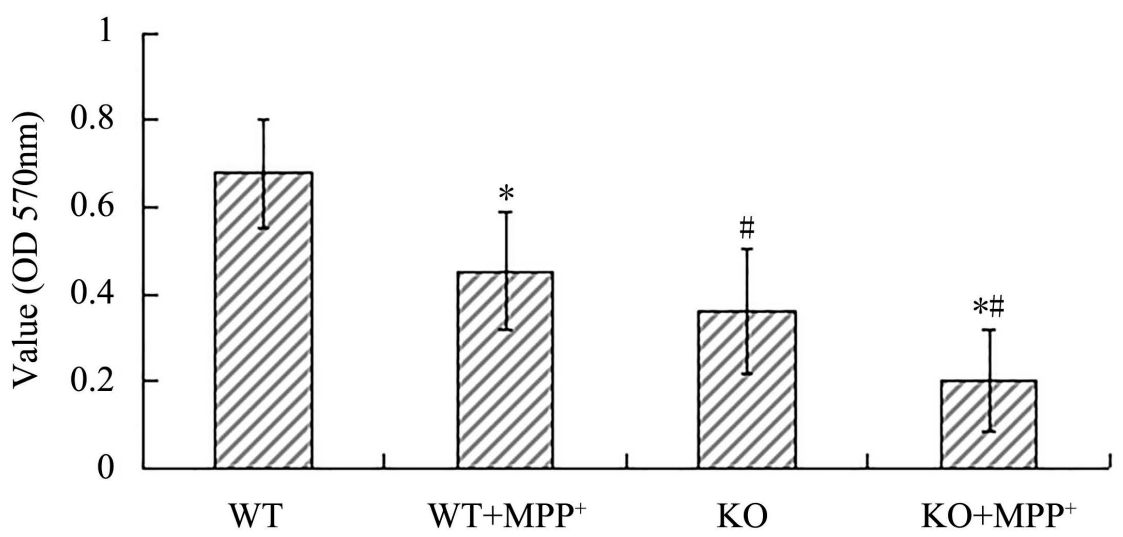

Figure 1. DJ-1 has an effect on cell survival. The collected cells are inoculated on 96-well culture plate for $6 \mathrm{~h}$ in $37^{\circ} \mathrm{C}$ and $50 \mathrm{ml} / \mathrm{LCO}_{2}$ incubator, $100 \mu \mathrm{l}$ culture medium per hole, including $1 \times 10^{3} \mathrm{PC} 12$ cells. Then removed the culture medium and intervened, the cells are divided into four groups according to the intervention conditions, that is, WT, WT + $\mathrm{MPP}^{+}, \mathrm{KO}$ and $\mathrm{KO}+\mathrm{MPP}^{+}$. The intervention group is added to the DMEM group with a final concentration of $5 \mu \mathrm{g} / \mathrm{mL} \mathrm{MPP}^{+}$. After $4 \mathrm{~h}, 10 \mu \mathrm{l} \mathrm{MTT}$ solution (final concentration $500 \mathrm{mg} / \mathrm{L}$, Biyuntian Biotechnology Research Institute) is added to each hole for $4 \mathrm{~h}$, in which 6 holes are set up in each group. Then, we add $150 \mu \mathrm{l}$ DMSO per hole in $37^{\circ} \mathrm{C}$, incubated until formazan crystals dissolve. Zero with a blank hole, the absorbance value of wavelength $570 \mathrm{~nm}(\mathrm{OD} 570 \mathrm{~nm}$ ) is measured by enzyme labeling instrument. Survival rate of PC-12 cells is expressed as OD $570 \mathrm{~nm}$. *: $\mathrm{p} \leq 0.05$ vs. control group; \#: $\mathrm{p} \leq 0.05$ vs. wild-type group. 
more sensitive to oxidative stress and easier to damage and DJ-1 has protective effect on oxidative stress.

The Cys-106 site of DJ-1 protein can undergo oxidative reaction under oxidative stress.

DJ-1 consists of 189 amino acids, of which the amino acid position 106 (Cys-106) is highly sensitive to oxidative stress, and the Cys-106 oxidation state determines the functional level of DJ-1 [13]. Therefore, the oxidation characteristics of different cells under oxidative stress must be observed first. In this study, we evaluate the oxidation of DJ-1 at Cys-106 sites (oxDJ-1) under oxidative stress. Through immunofluorescence microscopy, we find the absence of significant oxidation at the site of cys106 under physiological condition. Under $\mathrm{MPP}^{+}$-induced stress, oxDJ-1 increased significantly (Figure 2). Consistent with the results obtained by immunofluorescence microscopy, western blotting analysis clearly shows that the Cys-106 sites of DJ-1 proteins are oxidized in the state of oxidative stress, while PC-12 cells with DJ-1 knockout show no similar response.

The oxDJ-1 can bind directly to the C-Raf kinase domain, induce C-Raf phosphorylation at Ser-338 sites and promote C-Raf activation.

C-Raf can be activated by dephosphorylation of Ser-43,259 and 621 sites or by Ser-338 phosphorylation. Exposure to $\mathrm{MPP}^{+}$, whether does oxDJ-1 activate C-Raf? How does it activate C-Raf? In this study, the C-Raf protein of wild-type PC-12 cells is inactivated in base state. Exposed to $\mathrm{MPP}^{+}, \mathrm{C}-\mathrm{Raf}$ protein shows
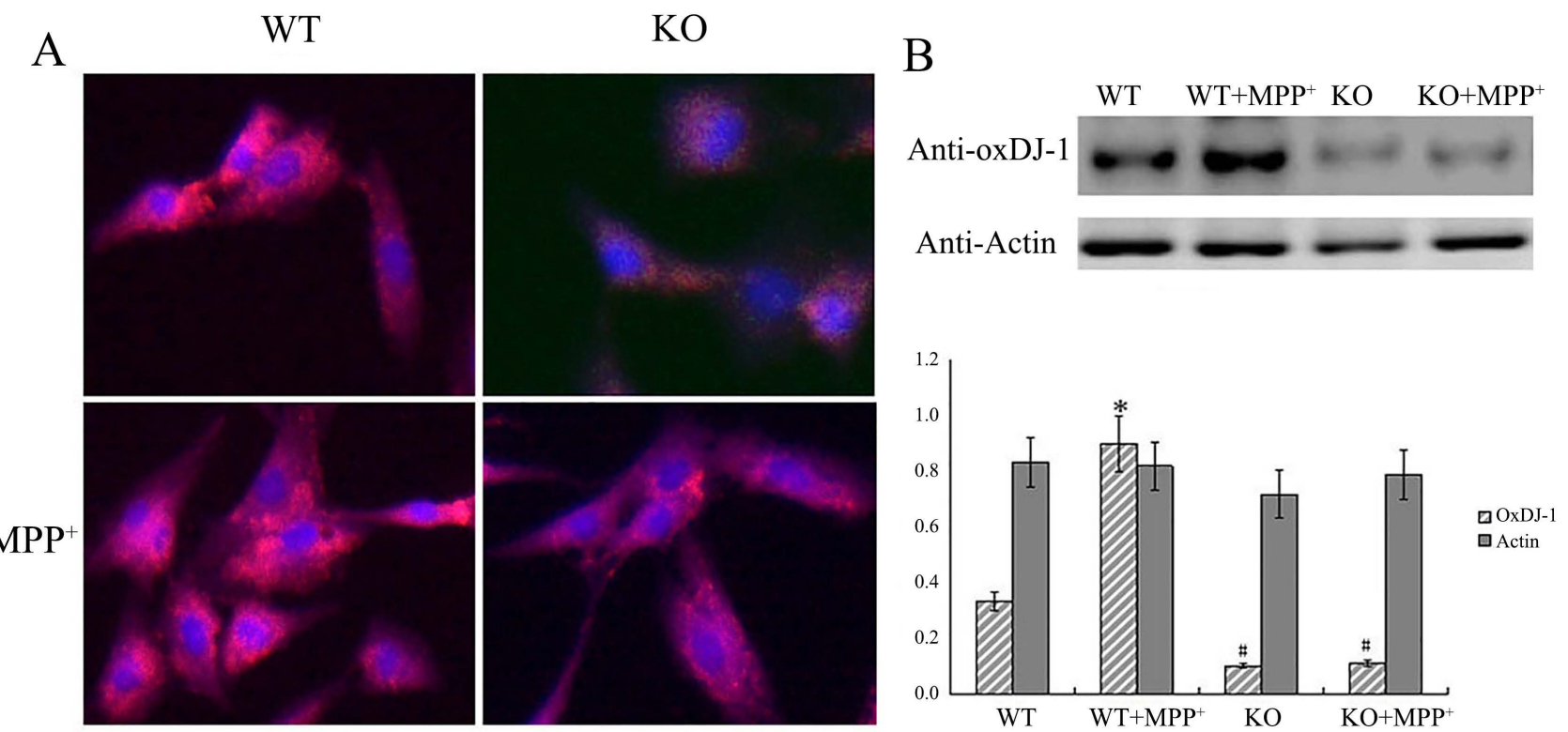

Figure 2. DJ-1 can be activated by oxidative stress at DJ-1 (Cys-106) sites. Wild type and DJ-1 knockout PC-12 cells are cultured in $0.1 \%$ calf serum medium at a density of $10^{3} / \mathrm{mL}$ for 6 hours. The cells are divided into 4 groups, that is, WT, WT $+\mathrm{MPP}+\mathrm{KO}^{+}$ and $\mathrm{KO}+\mathrm{MPP}^{+}$groups. The control groups only change the culture medium normally, and don't be added $\mathrm{MPP}^{+}, \mathrm{while}$ culture medium is added the $\mathrm{MPP}^{+}$(final concentration $5 \mathrm{ug} / \mathrm{mL}$ ) in intervention group for $30 \mathrm{~min}$. Then the cells are collected for the immunofluorescence observation. The antibodies used here are mouse anti-oxDJ-1 (cys-106) antibodies (1:1000, Abcam) and anti-mouse cy3 complement receptor monoclonal antibodies (1:200, Sigma). A: immunofluoresence $(\times 200)$; B: Western blotting analysis of oxDJ-1. *: $\mathrm{p} \leq 0.05$ vs. control group; \#: $\mathrm{p} \leq 0.05$ vs. wild-type group. 
obvious phosphorylation at Ser338 site and a little dephosphorylation at Ser259 site (Figure 2). But no such reaction is seen in DJ-1 knockout cells. Moreover, anti-oxDJ-1 antibodies are found to be confocal with C-Raf antibodies phosphorylated on Ser-338, but cannot confocal with C-Raf antibodies against Ser-259 dephosphorylation, suggesting that DJ-1 (Cys-106) activation in the cytoplasm directly causes C-Raf phosphorylation on the Ser-338 (Figure 3). Lack of DJ-1, oxidative stress is difficult to induce C-Raf activation. Although C-Raf can be activated by dephosphorylation of Ser259 sites, this activation is inadequate and cannot form complexes with activated DJ-1.

DJ-1 regulates activation and nuclear translocation of ERK signaling pathways through C-Raf activation.

OxDJ-1 binds to C-Raf and causes phosphorylation of Ser-338 sites, leading to C-Raf activation. What is the effect of activated complexes on MAPK/ERK signaling pathways? We culture wild-type and DJ-1 knockout PC-12 cells, divide them into control groups and intervention groups. The intervention groups are exposed to $\mathrm{MPP}^{+}$, and subsequently intervened with sorafenib and PD98059. The effects of DJ-1 and oxidative stress on MAPK/ERK signaling pathway are observed by immunofluorescence (Figure $4(\mathrm{~A})$ ) and western blotting (Figure $4(B)$, Figure $4(C)$ ), respectively. In the study, we first observe the changes in the level of intracellular pERK (Figure 4(B)). The results show that knockout DJ-1 can lead to an increase of intracellular pERK, and exposure to $\mathrm{MPP}^{+}$induces a more obvious increase, which reacts to significant differences. The exposure of

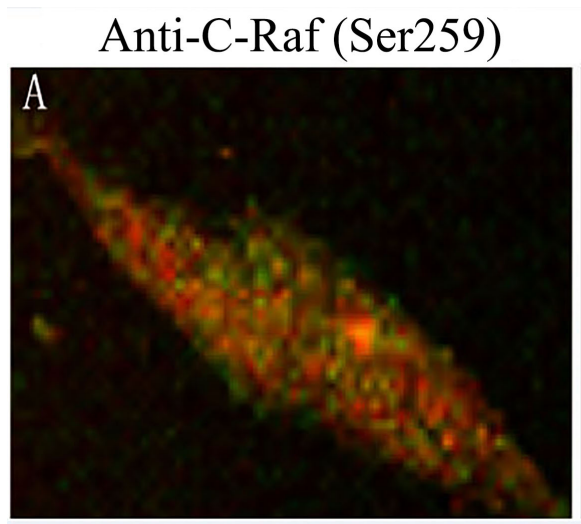

Anti-p-C-Raf (Ser338)

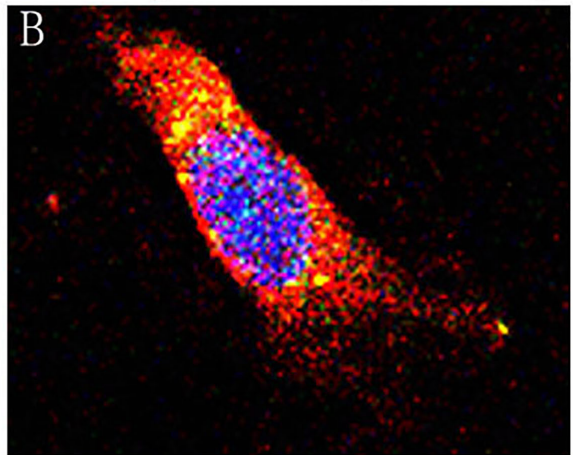

Figure 3. The oxDJ-1 induces C-Raf phosphorylation at Ser-338 sites. Wild type and DJ-1 knockout PC-12 cells are cultured in $0.1 \%$ calf serum medium at a density of $10^{3} / \mathrm{mL}$ for 6 hours. The cells are divided into 4 groups, that is, $\mathrm{WT}, \mathrm{WT}+\mathrm{MPP}^{+}, \mathrm{KO}$ and $\mathrm{KO}+\mathrm{MPP}^{+}$ groups. The control groups change the culture medium only normally, without $\mathrm{MPP}^{+}$. $\mathrm{MPP}^{+}$is added to the culture medium in the invention group (final concentration 5 $\mathrm{ug} / \mathrm{mL}$ ) for 2 hours. Cells collected are labeled with mouse anti phosphorylation C-Raf (Ser338, 1:1000, Biorbyt) monoclonal antibodies, mouse anti C-Raf (Ser259, 1:1000, Biorbyt) antibody or rabbit anti-oxDJ-1 polyclonal antibodies (1:1000, Abcam). Subsequently the cells were labeled with rabbit anti-Cy3 or mouse anti-complement monoclonal antibodies (1:200, Sigma). Observing under a laser confocal microscope $(\times 300)$, we find that DJ-1 co-located with p-C-Raf (Ser338) (B), showed bright yellow, and no confocal with C-Raf (Ser259) (A), still green fluorescence. 


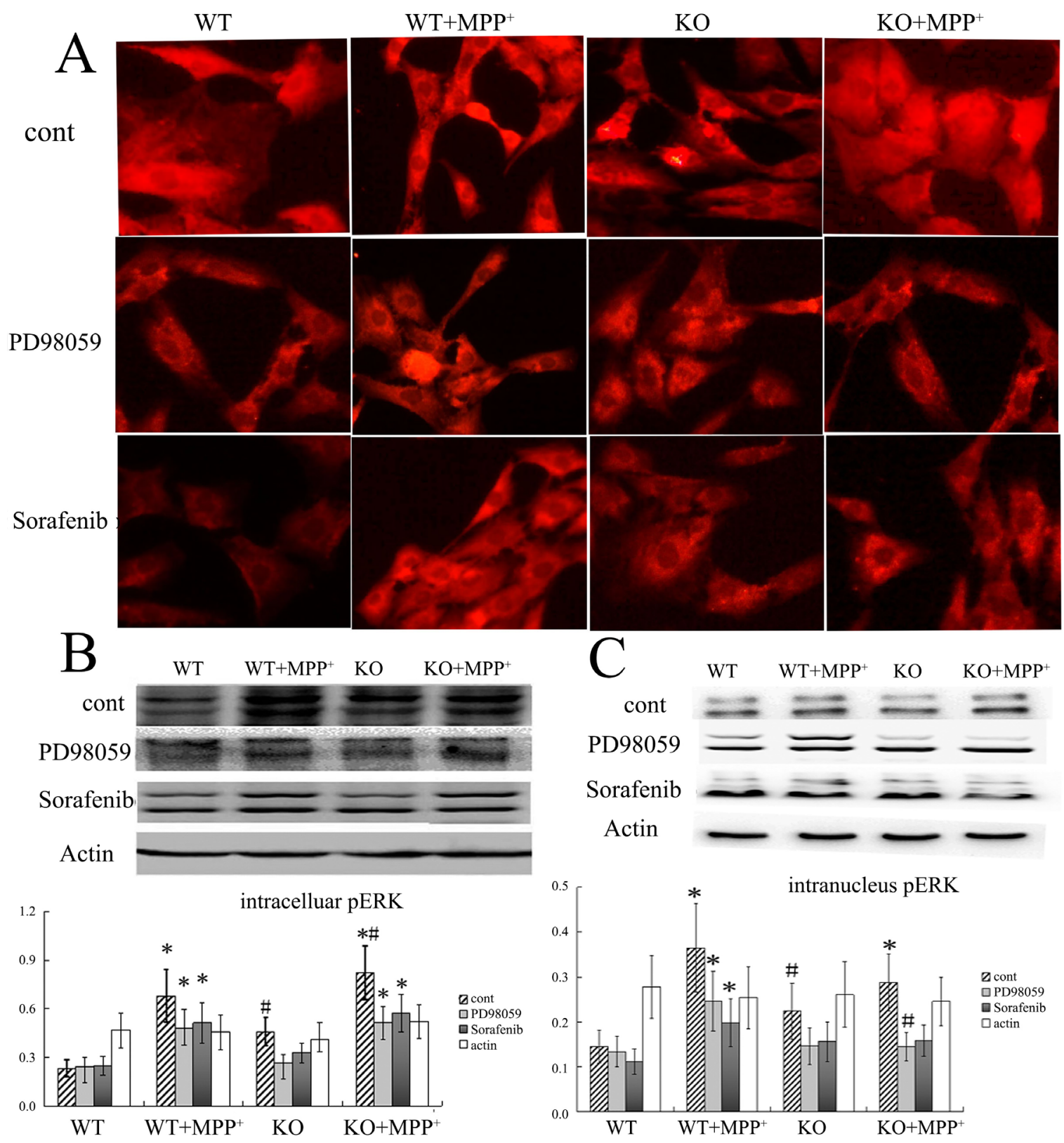

Figure 4. PD98059 and sorafenib have an effect on DJ-1 related pERK1/2. We culture PC-12 cells and DJ-1 knockout PC-12 cells at a density of $10^{2} / \mathrm{mL}$ for $24 \mathrm{~h}$. Then the cells are divided into three large groups and four subgroups, the four subgroups remain $\mathrm{WT}, \mathrm{WT}+\mathrm{MPP}^{+}, \mathrm{KO}$ and $\mathrm{KO}+\mathrm{MPP}^{+}$groups. They are cultured in $0.1 \%$ calf serum medium for $6 \mathrm{~h}$, added with $\mathrm{MPP}^{+}(5 \mathrm{ug} / \mathrm{mL})$ or not for $2 \mathrm{~h}$, which is the first group, and the second and third groups are preincubated with PD98059 $(10 \mu \mathrm{mol} / \mathrm{L})$ or sorafenib $(5 \mu \mathrm{mol} / \mathrm{L})$ for $20 \mathrm{~min}$, and then incubated with MPP $(5 \mu \mathrm{g} / \mathrm{mL})$ or for $2 \mathrm{~h}$. Respectively, then collecting cells for immunofluorescence. When the cells are cultured for western blotting, the inoculation density is $10^{6} / \mathrm{mL}$, in $75 \mathrm{~cm}^{2}$ culture bottles, and the total protein and nuclear protein of the cells are extracted according to the requirements of the protein extraction kit, then collecting proteins for western blotting. Anti pERK1/2, anti ERK1/2, and anti actin antibody are used to Western blotting. (A) Immunofluorescence $(\times 200)$; (B) Western blotting analyses of intracellular pERK; (C) Western blotting analyses of intranucleus pERK. *: p $\leq 0.05$ vs. control group; \#: $\mathrm{p} \leq 0.05$ vs. wild-type group.

DJ-1 knockout cells to $\mathrm{MPP}^{+}$caused further increase in intracellular pERK, but there isn't achieve significant difference. PD98059 can inhibit ERK activation 
caused by DJ-1 knockdown or $\mathrm{MPP}^{+}$exposure, as can sorafenib, but the degree of inhibition is lower than that of PD98059. Subsequently, we observed changes of pERK levels in the cell nucleus (Figure $4(C)$ ), and the results show that exposure to $\mathrm{MPP}^{+}$increases the level of wild-type cell nuclear $\mathrm{pERK}$, which reaches a significant difference, while knockout DJ-1 also increases the levels of intranucleus pERK, but there isn't significant difference. PD98059 or sorafenib can inhibit the increase of nuclear pERK, but only sorafenib inhibition to wild-type cell nuclear pERK is more obvious than PD98059, which achieves a significant difference.

\section{DJ-1 can improve cell survival by promoting autophagy maturation.}

We collect all kinds of experimental cells, process them according to the procedure of electron microscope observation, and observe the morphological characteristics of cells by transmission electron microscope. The results show that DJ-1 knockout cells have more autophagy stress than wild-type PC-12 cells in the base stage, and both wild-type PC-12 cells and DJ-1 knockout cells can induce stronger autophagy stress in the face of oxidative stress (Figure $5(A)$ ). Wild-type PC-12 cells can produce more autophagolysosomes (Figure 5(B)), while DJ-1 knockout cells are mainly autophagosomes (Figure 5(C)), and mitochondria are irregular. PD98059 can reduce autophagy stress in wild-type PC-12 cells under oxidative stress, and the number of autophagolysosomes is also reduced; while sorafenib cannot down-regulate autophagy stress in DJ-1 knockout cells, but the number of autophagolysosomes decrease more significantly in wild-type PC-12 cells (Figure 5(D)). PD98059 can reduce the degree of autophagical stress, and there is no significant change in the proportion of autophagolysosomes/autophagosomes. Sorafenib can also down-regulate the degree

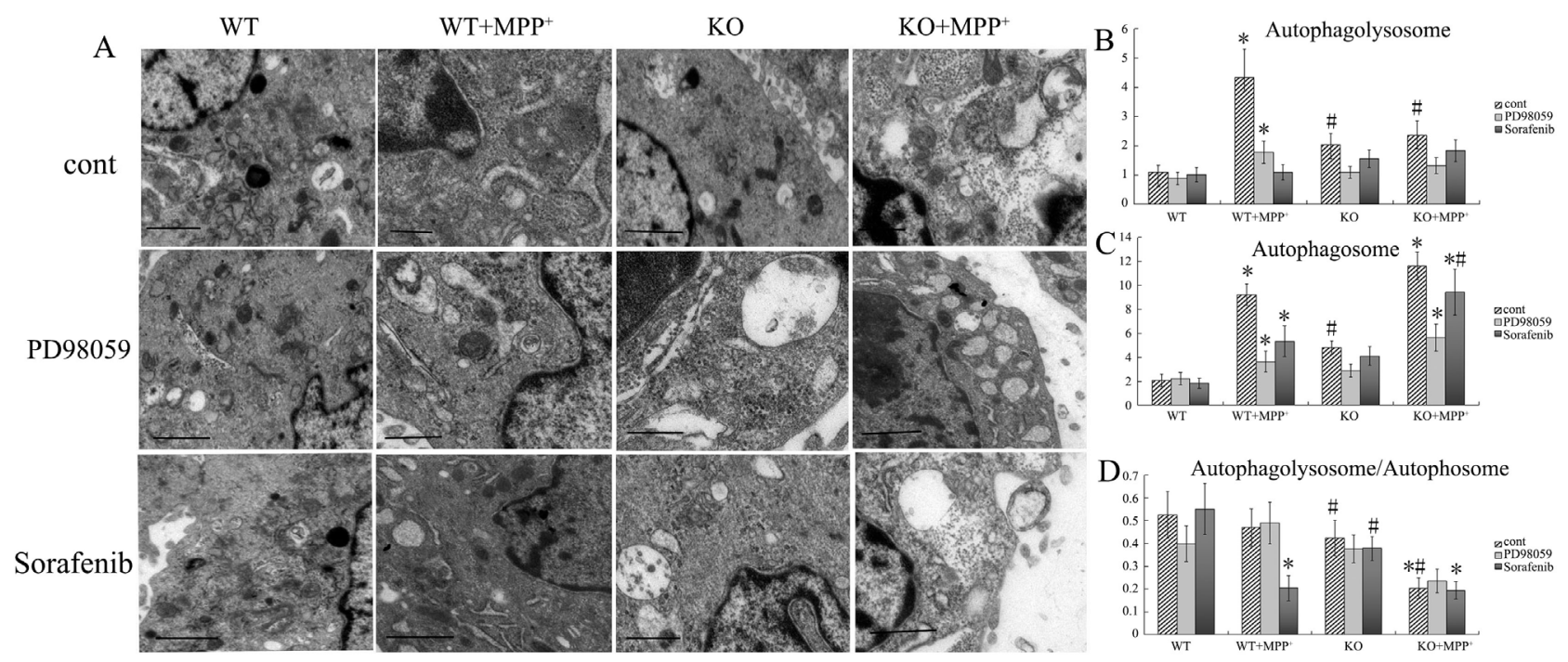

Figure 5. DJ-1 regulates autophagy stress through Ras/ERK. Under TEM, two cells are randomly selected to count the number of autophagosomes and autophagolysosomes. The average value is counted as one sample, and each group includes 5 samples. Finally, the ratio of autophagolysosomes to autophagosomes was obtained. The data were obtained in a double-blind way by two people alone. bar = $500 \mathrm{~nm}$. (A) Photo of TEM; (B) value of autophagolysosomes in a cell; (C) value of autophagosomes in a cell; (D) ratio of autophagolysosomes to autophagosomes in a cell. ${ }^{*}$ : $p \leq 0.05$ vs. control group; \#: $p \leq 0.05$ vs. wild-type group. 
of autophagy stress. For wild-type PC-12 cells, the proportion of autophagolysosomes/autophagosomes decreased significantly, while for DJ-1 knockout cells, the proportion of autophagolysosomes/autophagosomes remained basically unchanged, which suggests C-Raf play an important role in DJ-1 protection.

\section{Discussion}

Park 7 gene was found to have an autosomal recessive deletion mutation in 2003, leading to early onset Parkinson's disease (PD) [14]. DJ-1 is a multifunctional protein [15] [16] and plays an important role in antioxidant defense and protecting cells from oxidative stress. DJ-1 binds to a variety of proteins, including various transcription factors, as coactivators or co-inhibitors, regulating its target genes without direct binding to DNA, thus affecting various functions of cells. During cell cycle, DJ-1 shifts from cytoplasm to nucleus after mitogen stimulation [17], which plays an important role in antioxidant stress, transcriptional regulation, signal transduction pathway regulation, and chaperone and protease responses [14] [18] [19] [20]. In this study, in order to observe the effect of DJ-1 on cell survival, we start from the basic state to the oxidative stress state respectively, and found that DJ-1 knockout seriously affects the viability, which supports the above views.

C-Raf is a serine/threonine kinase that binds to GTP-RAS in its $\mathrm{N}$ terminal region [19] [21] [22]. C-Raf is phosphorylatedatSer43, 259 and 621 sites and deactivated in the absence of RAS signals; when some signals cause C-Raf to be dephosphorylated at Ser43,259 and 621 sites by protein phosphatase 2A, C-Raf is activated [1] [23] [6] [24]. The study also finds that when C-Raf is EGF phosphorylated at Ser-338 sites, C-Raf can be directly activated [15] [16]. OxDJ-1 also stimulates c-Raf autophosphorylation in vitro [25]. Whether oxDJ-1 can also activate C-Raf in vivo? In this study, we find that oxidative stress can promote the oxidation of Cys106 sites in DJ-1, thus activating DJ-1. Activated DJ-1 can directly lead to phosphorylation of C-Raf at Ser-338 sites and activate the C-Raf. DJ-1 knockout PC-12 cells have no DJ-1, so there is no activated DJ-1, which cannot lead to phosphorylation of C-Raf on the Ser-338, and it cannot activate the $\mathrm{C}$-Raf. The results suggest that oxidative stress can promote the activation of DJ-1, subsequently induce C-Raf phosphorylation at Ser-338 sites. Although oxidative stress also leads to dephosphorylation of C-Raf at Ser259 sites, it is less numerous and cannot be confocal with activated DJ-1, suggesting that oxDJ-1 activate C-Raf pathway mainly through phosphorylation at Ser338 sites rather than dephosphorylation at Ser259 sites

OxDJ-1 acts directly on C-Raf by promoting C-Raf phosphorylation at Ser338 sites subsequently activates MEK/ERK signaling pathways (Figure 6). But how does it regulate downstream MAPK/ERK signaling pathways? What are the characteristics? In this study, we also find that exposure to $\mathrm{MPP}^{+}$, wild-type $\mathrm{PC}-12$ cells can rapidly promote phosphorylation of the ERK pathway, and DJ-1 knockdown cells have higher phosphorylation levels in the pathway. PD98059 


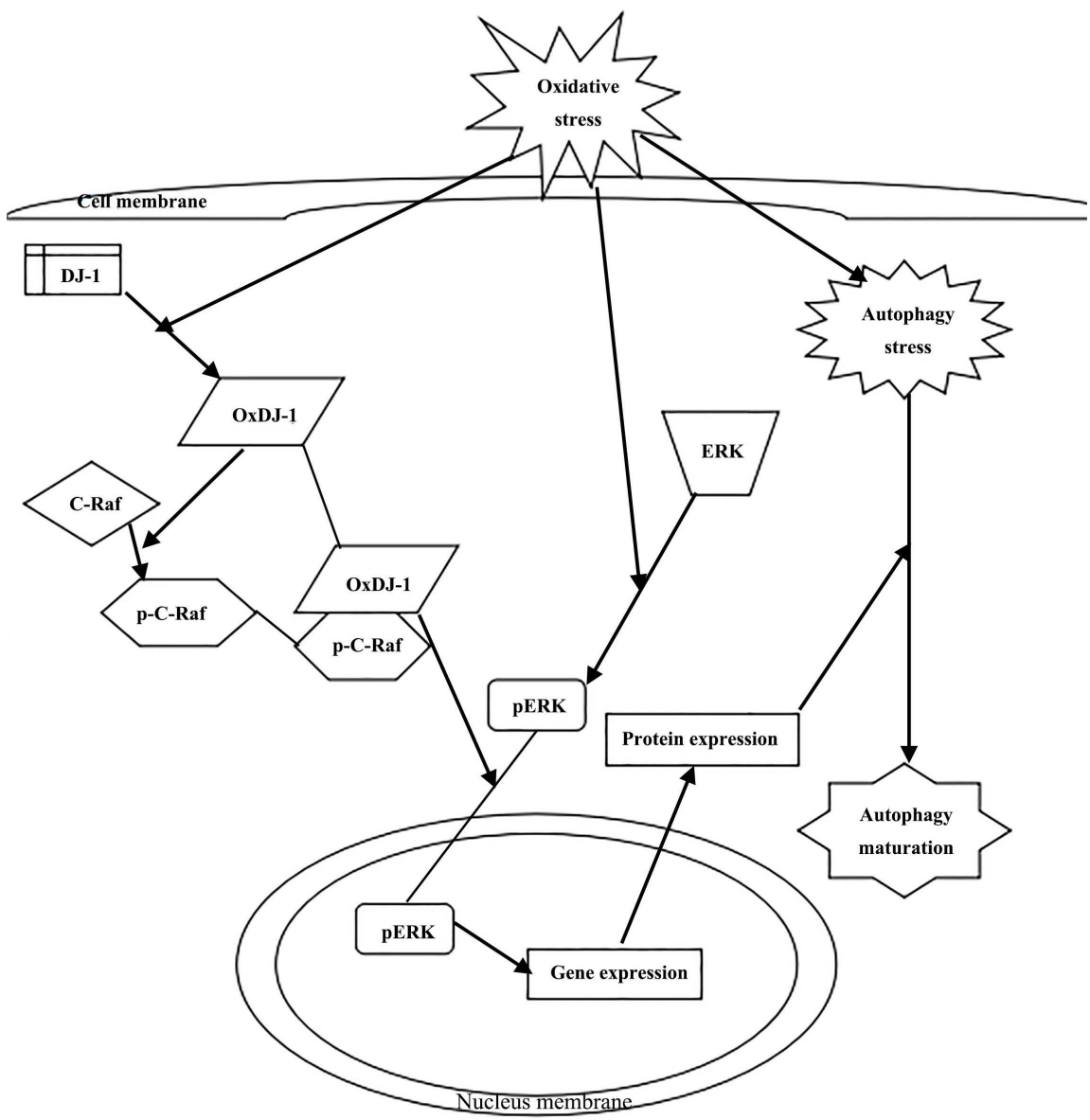

Figure 6. Schematic figure of DJ-1 in Raf/ERK pathways. Oxidative stress leads to DJ-1 activation by oxidation at Cys106 sites while stimulating intracellular ERK kinase phosphorylation. The cross-talk between the oxDJ-1 and C-Raf induces phosphorylation of C-Raf at Ser338 sites leads to C-Raf activation, which forms a complex with oxDJ-1. The complex promotes pERK nuclear translocation, subsequently regulates the expression of related genes. The genes regulate the expression of proteins, which promotes autophagosomes to autophagolysosomes transformation and induces autophagy maturation.

can inhibit the activation and nuclear translocation of ERK signaling pathways, and the degree of inhibition is almost similar. In wild-type cells, sorafenib can slightly down-regulate ERK1/2 phosphorylation, but it can significantly inhibit nuclear translocation, which doesn't exist for DJ-1knockout cells, suggesting that $\mathrm{C}$-Raf activation plays a key role in the DJ-1 regulation of signaling pathways. C-Raf (Ser338) site phosphorylation activates the MEK/ERK signaling pathway, which phosphorylates the ERK1/2 [26]. The activated p-ERK1/2 eventually transfers from the cytoplasm to the nucleus, and pERK1/2 phosphorylates related transcription factors and promotes cell growth or autophagy maturation [25] [27] [28]. Under oxidative stress, the wild-type PC-12 cells show this feature better, but for DJ-1 knockout PC-12 cells, despite some degree of activation of ERK pathways, pERK1/2 nuclear translocation was reduced. Oxidative stress promotes autophagy stress in wild-type PC-12 cells, leading to increased autophagy, as well as for DJ-1 knockout PC-12 cells. Moreover, the inhibition of 
C-Raf activation can reduce the degree of autophagy stress, and the proportion of autophagolysosome/autophagosome decreases, which inhibits the maturation of autophagy. Inhibition of ERK signaling pathways, whether wild-type DJ-1 cells or DJ-1 knockout cells, reduced the extent of autophagical stress, but did not change the proportion of autophagolysosomes/autophagosomes. Therefore, we have reason to speculate that DJ-1 could promote autophagy maturation through C-Raf/ERK signal pathway thus improving cell survival (Figure 6).

\section{Acknowledgements}

This work was funded by the Zhenjiang Social Development Project (SH2018026) of Jiangsu, China.

\section{Conflicts of Interest}

The authors declare no conflicts of interest regarding the publication of this paper.

\section{References}

[1] Xue, R., Jiang, J., Dong, B., Tan, W., Sun, Y., Zhao, J., Chen, Y., Dong, Y. and Liu, C. (2017) DJ-1 Activates Autophagy in the Repression of Cardiac Hypertrophy. Archives of Biochemistry and Biophysics, 633, 124-132. https://doi.org/10.1016/j.abb.2017.09.012

[2] Gómez-Sánchez, R., Yakhine-Diop, S.M., Bravo-San Pedro, J.M., Pizarro-Estrella, E., Rodríguez-Arribas, M., Climent, V., et al. (2015) PINK1 Deficiency Enhances Autophagy and Mitophagy Induction. Molecular \& Cellular Oncology, 3, Artocle ID: e1046579. https://doi.org/10.1080/23723556.2015.1046579

[3] Kobayashi, K., Liu, Y., Ichikawa, H., Takemura, S. and Minamiyama, Y. (2020) Effects of Bisphenol A on Oxidative Stress in the Rat Brain. Antioxidants, 9, Article No. 240. https://doi.org/10.3390/antiox9030240

[4] Yeo, H., Yeo, E.J., Shin, M.J., Choi, Y.J., Lee, C.H., Kwon, H.Y., Kim, D.W., Eum, W.S. and Choi, S.Y. (2018) Protective Effects of Tat-DJ-1 Protein against Streptozotocin-Induced Diabetes in a Mice Model. BMB Reports, 51, 362-367. https://doi.org/10.5483/BMBRep.2018.51.7.101

[5] Komatsu, M., Waguri, S., Chiba, T., Murata, S., Iwata, J., Tanida, I., et al. (2006) Loss of Autophagy in the Central Nervous System Causes Neurodegeneration in Mice. Nature, 441, 880-884. https://doi.org/10.1038/nature04723

[6] Degirmenci, U., Wang, M. and Hu, J. (2020) Targeting Aberrant RAS/RAF/MEK/ERK Signaling for Cancer Therapy. Cells, 9, Article No. 198. https://doi.org/10.3390/cells9010198

[7] Corcelle, E., Nebout, M., Bekri, S., Gauthier, N., Hofman, P., Poujeol, P., Fénichel, P. and Mograbi, B. (2006) Disruption of Autophagy at the Maturation Step by the Carcinogen Lindane Is Associated with the Sustained Mitogen-Activated Protein Kinase/Extracellular Signal-Regulated Kinase Activity. Cancer Research, 66, 6861-6870. https://doi.org/10.1158/0008-5472.CAN-05-3557

[8] Li, X.Z., Sui, C.Y., Chen, Q., Chen, X.P., Zhang, H. and Zhou, X.P. (2015) Upregulation of Cell Surface Estrogen Receptor Alpha Is Associated with the Mitogen-Activated Protein Kinase/Extracellular Signal-Regulated Kinase Activity and Promotes Autophagy Maturation. International Journal of Clinical and Experimen- 
tal Pathology, 8, 8832-8841.

[9] Tong, Y., Huang, H. and Pan, H. (2015) Inhibition of MEK/ERK Activation Attenuates Autophagy and Potentiates Pemetrexed-Induced Activity against HepG2 Hepatocellular Carcinoma Cells. Biochemical and Biophysical Research Communications, 456, 86-91. https://doi.org/10.1016/j.bbrc.2014.11.038

[10] Salthun-Lassalle, B., Hirsch, E.C., Wolfart, J., Ruberg, M. and Michel, P.P. (2004) Rescue of Mesencephalic Dopaminergic Neurons in Culture by Low-Level Stimulation of Voltage-Gated Sodium Channels. Journal of Neuroscience, 24, 5922-5930. https://doi.org/10.1523/JNEUROSCI.5668-03.2004

[11] Mograbi, B., Corcelle, E., Defamie, N., Samson, M., Nebout, M., Segretain, D., Fe'nichel, P. and Pointis, G. (2003) Aberrant Connexin 43 Endocytosis by the Carcinogen Lindane Involves Activation of the ERK/Mitogen-Activated Protein Kinase Pathway. Carcinogenesis, 24, 1415-1423. https://doi.org/10.1093/carcin/bgg093

[12] Le'Negrate, G., Selva, E., Auberger, P., Rossi, B. and Hofman, P. (2000) Sustained Polymorphonuclear Leukocyte Transmigration Induces Apoptosis in T84 Intestinal Epithelial Cells. Journal of Cell Biology, 150, 1479-1488.

https://doi.org/10.1083/jcb.150.6.1479

[13] Oh, S.E., Park, H.J., He, L., Skibiel, C., Junn, E. and Mouradian, M.M. (2018) The Parkinson's Disease Gene Product DJ-1 Modulates miR-221 to Promote Neuronal Survival against Oxidative Stress. Redox Biology, 19, 62-73.

https://doi.org/10.1016/j.redox.2018.07.021

[14] Antipova, D. and Bandopadhyay, R. (2017) Expression of DJ-1 in Neurodegenerative Disorders. In: Ariga, H. and Iguchi-Ariga, S., Eds., DJ-1/PARK7 Protein, Vol. 1037, Springer, Singapore, 25-43. https://doi.org/10.1007/978-981-10-6583-5_3

[15] Luo, F., Shu, M., Gong, S., Wen, Y., He, B., Su, S. and Li, Z. (2019) Antiapoptotic Activity of Chlamydia trachomatis Pgp3 Protein Involves Activation of the ERK1/2 Pathway Mediated by Upregulation of DJ-1 Protein. Pathogens and Disease, 77, ftaa003. https://doi.org/10.1093/femspd/ftaa003

[16] Ahn, S., Liu, Q.F., Jang, J.H., Park, J., Jeong, H.J., Kim, Y., et al. (2019) Gami-Chunggan Formula Prevents Motor Dysfunction in MPTP/p-Induced and A53T alpha-Synuclein Overexpressed Parkinson's Disease Mouse Model Though DJ-1 and BDNF Expression. Frontiers in Aging Neuroscience, 11, Article No. 230. https://doi.org/10.3389/fnagi.2019.00230

[17] Takahashi-Niki, K., Niki, T., Iguchi-Ariga, S.M.M. and Ariga, H. (2017) Transcriptional Regulation of DJ-1. In: Ariga, H. and Iguchi-Ariga, S., Eds., DJ-1/PARK7 Protein, Vol. 1037, Springer, Singapore, 89-95. https://doi.org/10.1007/978-981-10-6583-5_7

[18] Meijer, A.J. and Codogno, P. (2007) Macroautophagy: Protector in the Diabetes drama? Autophagy, 3, 522-525. https://doi.org/10.4161/auto.4449

[19] Milisav, I., Šuput, D. and Ribarič, S. (2015) Unfolded Protein Response and Macroautophagy in Alzheimer's, Parkinson's and Prion Diseases. Molecules, 20, 22718-22756. https://doi.org/10.3390/molecules201219865

[20] Oh, S.E. and Mouradian, M.M. (2018) Cytoprotective Mechanisms of DJ-1 against Oxidative Stress through Modulating ERK1/2 and ASK1 Signal Transduction. Redox Biology, 14, 211-217. https://doi.org/10.1016/j.redox.2017.09.008

[21] Xu, C.Y., Kang, W.Y., Chen, Y.M., Jiang, T.F., Zhang, J., Zhang, L.N., et al. (2017) DJ-1 Inhibits a-Synuclein Aggregation by Regulating Chaperone-Mediated Autophagy. Frontiers in Aging Neuroscience, 27, Article No. 308. https://doi.org/10.3389/fnagi.2017.00308 
[22] Inden, M., Yanagisawa, D., Hijioka, M., Ariga, H. and Kitamura, Y. (2017) Therapeutic Activities of DJ-1 and Its Binding Compounds against Neurodegenerative Diseases. In: Ariga, H. and Iguchi-Ariga, S., Eds., DJ-1/PARK7 Protein, Vol. 1037, Springer, Singapore, 187-202. https://doi.org/10.1007/978-981-10-6583-5_12

[23] Nash, Y., Schmukler, E., Trudler, D., Pinkas-Kramarski, R. and Frenkel, D. (2017) DJ-1 Deficiency Impairs Autophagy and Reduces Alpha-Synuclein Phagocytosis by Microglia. Journal of Neurochemistry, 143, 584-594.

https://doi.org/10.1111/jnc. 14222

[24] Akula, S.M., Abrams, S.L., Steelman, L.S, Emma, M.R., Augello, G., Cusimano, A., et al. (2019) RAS/RAF/MEK/ERK, PI3K/PTEN/AKT/mTORC1 and TP53 Pathways and Regulatory miRs as Therapeutic Targets in Hepatocellular Carcinoma. Expert Opinion on Therapeutic Targets, 23, 915-929.

https://doi.org/10.1080/14728222.2019.1685501

[25] Takahashi-Niki, K., Kato-Ose, I., Murata, H., Maita, H. and Iguchi-Ariga, S.M.M. (2015) Epidermal Growth Factor-dependent Activation of the Extracellular Signal-Regulated Kinase Pathway by DJ-1 Protein. The Journal of Biological Chemistry, 290, 17838-17847. https://doi.org/10.1074/jbc.M115.666271

[26] Nichols, R.J., Haderk, F., Stahlhut, C., Schulze, C.J., Hemmati, G., Wildes, D., et al. (2018) RAS Nucleotide Cycling Underlies the SHP2 Phosphatase Dependence of Mutant BRAF-, NF1- and RAS-Driven Cancers. Nature Cell Biology, 20, 1064-1073. https://doi.org/10.1038/s41556-018-0169-1

[27] Guo, Y.J., Pan, W.W., Liu, S.B., Shen, Z.F., Xu, Y. and Hu, L.L. (2020) ERK/MAPK Signalling Pathway and Tumorigenesis. Experimental and Therapeutic Medicine, 19, 1997-2007. https://doi.org/10.3892/etm.2020.8454

[28] Li, X.Z., Sui, C.Y., Chen, Q., Zhuang, Y.S., Zhang, H. and Zhou, X.P. (2016) The Effects and Mechanism of Estrogen on Rats with Parkinson's Disease in Different Age Groups. American Journal of Translational Research, 8, 4134-4146. 